seen before - images that delight us as much for their beauty as for their informative power.

\section{Selected references}

1 O'Rourke, N. A. and Fraser, S. E. (1986) Dev. Biol. 114, 265276

2 Purves, D. and Voyvodic, J. T. (1987) Trends Neurosci. 10, 398-404

3 Lichtmann, J. W., Magrassi, L. and Purves, D. (1987) J. Neurosci. 7, 1215-1222

4 Katz, L. C. (1987) J. Neurosci. 7, 1223-1249

5 Cohen, L. B. and Salzberg, B. M. (1978) Rev. Physiol. Biochem. Pharm. 83, 85-88

6 Grinvald, A., Fine, A., Farber, I. C. and Hildesheim, R. (1983) Biophys. J. 42, 195-198

7 Grinvald, A. (1985) Annu. Rev. Neurosci. 8, 263-305

8 London, J. A., Zecevic, D., Loew, L. M., Orbach, H. S. and Cohen, L. B. (1986) in Applications of Fluorescence in the Biomedical Sciences (Taylor, D. L. et al., eds), pp. 423-437, Alan R. Liss

9 Williams, D. A., Fogarty, K. E., Tsien, R. Y. and Fay, F. S. (1985) Nature 318, 558-561

10 Ross, W. N., Lewenstein-Stockbridge, L. and Stockbridge,
N. L. (1986) J. Neurosci. 6, 1148-1159

11 Blasdel, G. G. and Salama, G. (1986) Nature 321, 579-585

12 Grinvald, A., Lieke, E., Frosti, R. D., Gilbert, C. D. and Wiesel, T. N. (1986) Nature 324, 361-364

13 Kauer, J. S. (1988) Nature 331, 166-168

14 Breuer, A. C., Allen, R. D. and Lewis, L. 1. (1981) Neurology 31, 118

15 Allen, R. D. (1985) Annu. Rev. Biophys. Chem. 14, 265-290

16 Inoué, S. (1986) Video Microscopy, Plenum Press

17 Agard, D. A. and Sedat, J. W. (1983) Nature 302, 676-681

18 Hiraoka, Y., Sedat, J. W. and Agard, D. A. (1987) Science 238, 36-41

19 Minsky, M. (1957) US Patent No. 3013467

20 Egger, M. D. and Petran, M. (1967) Science 157, 305-307

21 Boyde, A. (1985) Science 230, 1270-1272

22 Brakenhoff, G. J., van der Voort, H. T. M., van Spronsen, E. A., Linnemans, W. A. M. and Nanninga, N. (1985) Nature 317, 748-749

23 Carlsson, K. et al. (1985) Opt. Lett. 10, 53-55

24 White, J. G., Amos, W. B. and Fordham, M. (1987) J. Cell Biol. 105, 41-48

25 Grinvald, A., Anglister, L., Freeman, J. R., Hildesheim, R, and Manker, A. (1984) Nature 308, 848-850

26 Tsien, R. Y. (1981) Nature 290, 527-528 of object and background motion

\author{
Martin Egelhaaf, Klaus Hausen, Werner Reichardt, and Christian Wehrhahn
}

The spatial distribution of light intensity received by the eyes changes continually when an animal moves around in its environment. These retinal activity patterns contain a wealth of information on the structure of the environment, the direction and speed of selfmotion, and on the independent motion of objects ${ }^{1,2}$. If evaluated properly by the nervous system this information can be used in visual orientation. In a combination of both behavioural and electrophysiological analysis and modelling, this article establishes the neural mechanisms by which the visual system of the fly evaluates two types of basic retinal motion patterns: coherent retinal large-field motion as induced by selfmotion of the animal, and relative motion between objects and their background. Separate neuronal networks are specifically tuned to each of these motion pattems and make use of them in two different visual orientation tasks.

Visual orientation greatly relies on the evaluation of the global visual motion patterns received by the eyes when an animal moves around. These motion patterns depend in a characteristic way on the trajectory described by the moving animal as well as on the particular three-dimensional structure of the visual environment ${ }^{1,2}$. Consider, for instance, two simple commonplace situations. In the first, a moving animal unintentionally deviates from its course. This results in a displacement of the entire visual scene, which contains a strong rotational component. When this rotational component is extracted from the retinal motion pattern, it can be used to control the compensatory optomotor responses of the animal. In this way, the course may be stabilized against internal and external disturbances. A different situation is encountered when the animal passes nearby objects located in front of a more distant background. The retinal images of these objects and their background then move relative to each other leading to discontinuities in the motion field. This relative motion may indicate the existence of nearby stationary or moving objects. This information can be used to discriminate objects from their background and might serve as the basic cue in various visual orientation tasks, such as fixation of stationary objects or pursuit of moving targets. These types of global retinal motion patterns do not only occur when the animal moves around bodily. Similar motion patterns may also arise during head and eye movements.

This review concentrates on recent studies on the visual system of the fly, which has proved, during the past few decades, to be a suitable model system for the elucidation of the neuronal computations underlying various behavioural motion-dependent tasks ${ }^{3,4}$. We analyse the basic mechanisms by which the nervous system of the fly processes coherent largefield motion, and relative motion between objects and background, and how these motion patterns are exploited in mediating optomotor course stabilization and object-induced orientation. Whether related mechanisms play a role in evaluating global retinal motion patterns in other species has yet to be established, although it is not unreasonable to expect that this will be the case. This has already been shown for the mechanisms underlying other motion information processing tasks. The basic mechanism of local movement detection for instance (see below), which was initially discovered in the insect visual system, was later also found in vertebrates, including $\operatorname{man}^{5-8}$.
Martin Egelhaaf, Klaus Hausen, Werner Reichardt and Christian Wehriahn are at the MaxPlanck-Institut für biologische Kybernetik Spemannstrasse 38 , D-7400 Tübingen, FRG 
Local movement evaluation is necessary to detect coherent background motion, and relative motion between objects and background

The direction and velocity of a moving stimulus are not encoded explicitly at the level of the photoreceptor output. Instead, each photoreceptor provides information only on time-dependent variations of local light intensity. From these signals, the visual system computes perceived motion and other information. There is now good evidence that, in both the insect ${ }^{4-6}$ and human visual system ${ }^{7,8}$, the initial computation of motion is performed by local "elementary movement detectors' (EMDs), which are organized in twodimensional retinotopic arrays and cover the entire visual field. In principle, an EMD can be composed of two mirror-symmetrical subunits each of which evaluates a kind of spatiotemporal cross-correlation of the light intensity fluctuations at two neighbouring points in visual space. The final output of the detector is given by the difference between the outputs of the two subunits (Fig. 1A). An individual EMD of this kind is not a pure velocity sensor that correctly indicates the direction and velocity of local motion. Instead, its response is strongly influenced by the textural properties of the moving pattern such as its spatial frequency content and contrast ${ }^{4-6,9,10}$.
Since an EMD is oriented in space and thus has a particular preferred direction, differently oriented EMDs are required at each retinal location to obtain a two-dimensional representation of motion. The output of an array of orthogonally oriented pairs of EMDs is illustrated for two different motion patterns by the computer simulations shown in Fig. 1. The array is stimulated by the coherent movement of a large pattern and by relative movement of this pattern and an object having the same structure. The instantaneous activity of each pair of EMDs is represented as a vector indicating the local motion measurement in terms of direction and velocity. It is important to note that under both stimulus conditions the local response vectors, in general, do not coincide with the true directions of pattern motion. Depending on the local structure of the pattern, they may deviate from this direction by more than $90^{\circ}$. This demonstrates that local motion measurements by EMDs do not yield reliable information on the direction and velocity in which the different segments of the retinal image are moving. Thus, further processing steps are required to extract meaningful information from visual input.

How information on coherent large-field motion and relative motion is extracted from the ambiguous local motion measurements has been studied at different levels in flies; studies of visual orientation behaviour in

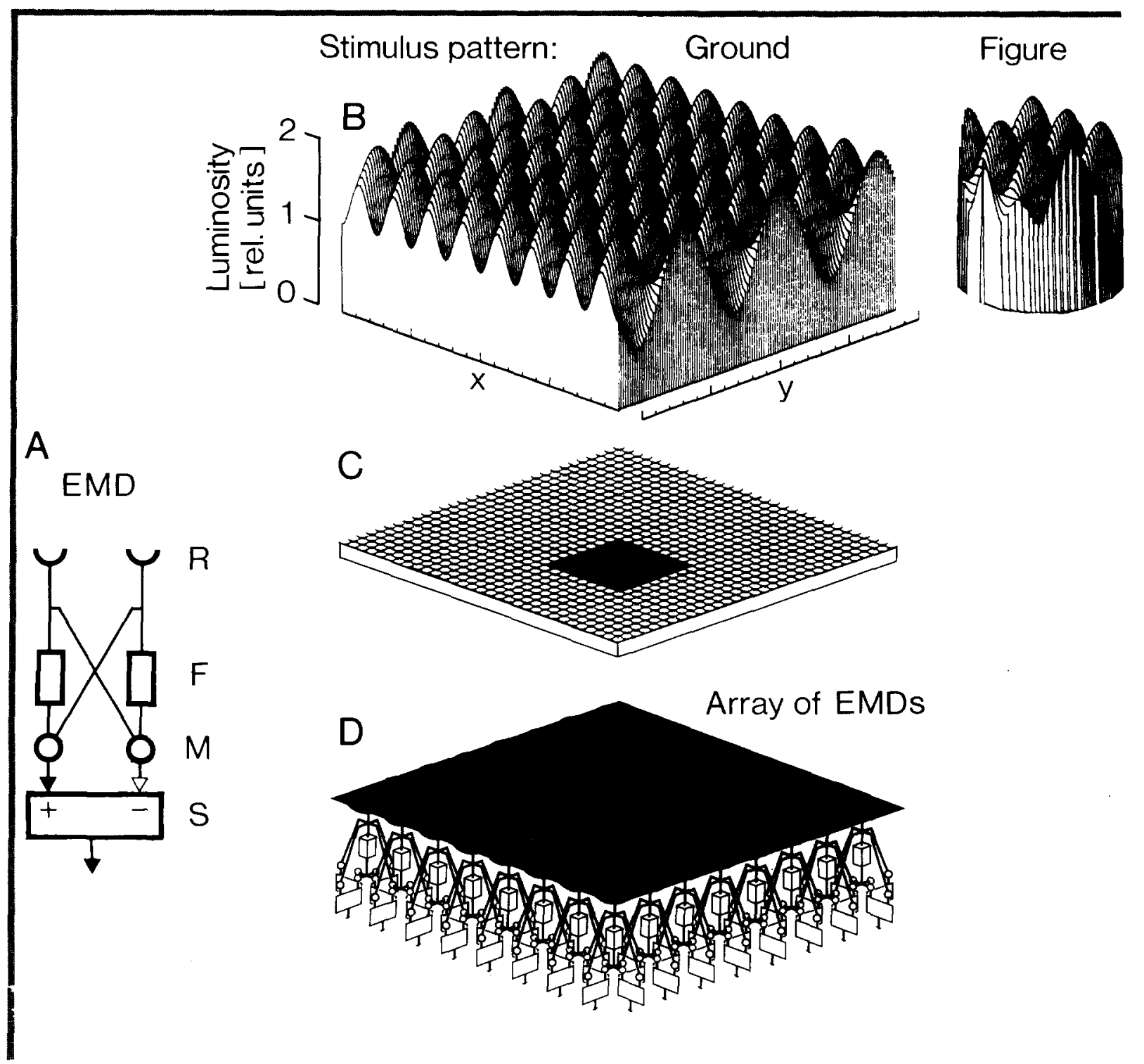


free $^{11-16}$ and tethered flight ${ }^{17-22}$, as well as of the response properties of visual interneurones ${ }^{20,23-27}$ have been carried out. However, only in female houseflies (Musca domestica) and blowflies ( $\mathrm{Cal}$ liphora erythrocephala), which have similar visual orientation responses, is a coherent view on how these tasks might be accomplished now emerging at both the behavioural and neuronal level. Therefore, we concentrate mainly on these species. Our studies started out from a quantitative behavioural analysis of stimulus-response relationships that formed the conceptual background of our neurophysiological

\section{Coherent motion of figure and ground}

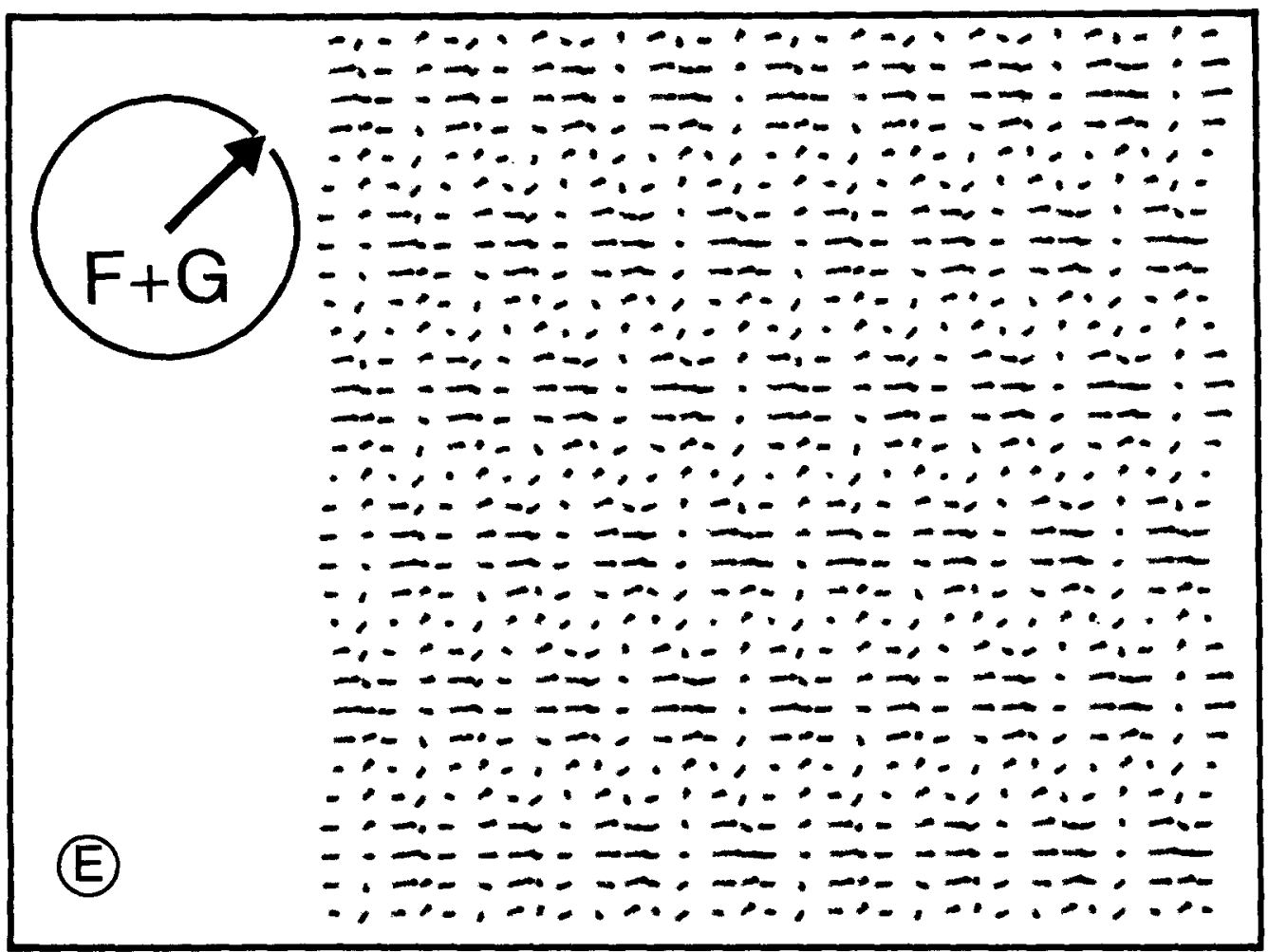

\section{Relative motion of figure and ground}

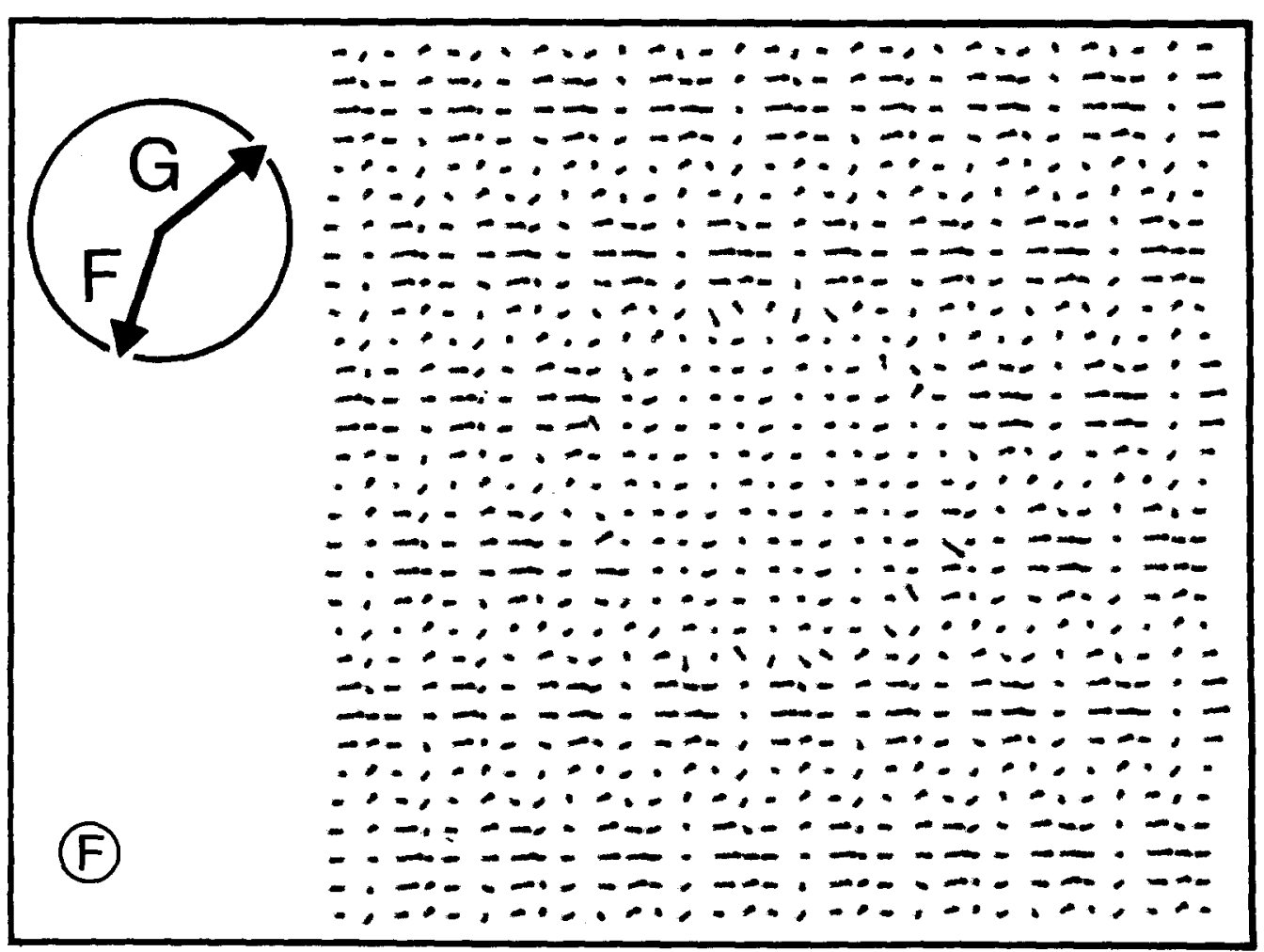

Fig. 1. Response pattern of a twodimensional array of elementary movement detectors (EMDs) under stimulation with coherent large-field and relative motion. (A) Schematic representation of a single EMD consisting of two mirror-symmetrical subunits. It receives input from two neighbouring photoreceptors $(R)$. A low-pass filter $(F)$ delays the signal in each branch, which is then multiplied (M) with the instantaneous signal of the neighbouring input channel. The difference (S) between the outputs of the two subunits gives the final detector output. Since an EMD receives input from two receptors, it is oriented in space. Therefore, a pair of differently oriented EMDs is required at each retinal location to obtain a two-dimensional representation of the local movement vector. (B) $A$ two-dimensional stimulus pattern consisting of a background pattern (ground) and a circular identically textured object (figure). There are two stimulus conditions: coherent movement of the ground and relative movement between figure and ground. In the latter condition, the central part of the pattern is considered as figure. (C) The stimulus pattern is seen by a square array of 961 pairs of EMDs. (D) Part of this array (blue) in greater detail. For convenience, the EMDs of each pair are assumed to be oriented orthogonally. (E) and (F) show the output of the entire array under two stimulus conditions. The output of each pair of EMDs at a given time is represented by a vector indicating the direction and amplitude of the local motion measurement. The activity distribution of the detector array is shown in two colours: orange indicates directions of the response vectors that deviate from the true direction of pattern motion by less than $\pm 90^{\circ}$; blue indicates deviations of the response vectors of more than $\pm 90^{\circ}$. In (E) figure (F) and ground (G) are moved coherently in only one direction. In (F) they move with the same velocity in different directions. Under both stimulus conditions the local response vectors, in general, do not coincide with the true direction of motion of the respective pattern segment. Depending on the local texture of the pattern they may even deviate from this direction by more than $90^{\circ}$. (Model adapted from Ref. 6.) 

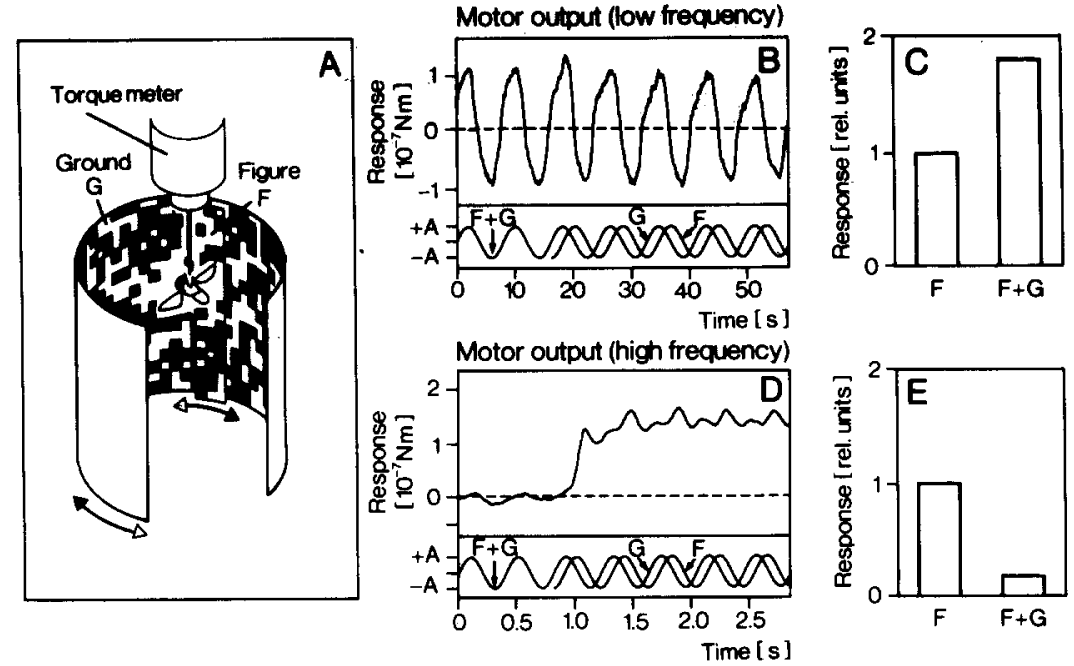

Fig. 2. Yaw torque responses to coherent and relative motion of figure and ground. (A) Experimental condition: the test fly is fixed to a torque meter that measures the fly's turning tendency as it flies. The fly is surrounded by a cylindrical panorama (ground, G) with a random texture; the cylinder has been opened to show the inside of the stimulus set-up. A vertically oriented textured stripe (figure, $F$ ) is placed in front of the ground. Its mean angular position was in front of the right eye $30^{\circ}$ from the frontal midline of the cylinder. Its angular width was $12^{\circ}(B)$ and $(D)$ or $10^{\circ}(C)$ and $(E)$. Both figure and ground could be moved horizontally either together or relative to each other. $\ln (\mathrm{B})$ and $(\mathrm{C})$ the pattern was oscillated at a low frequency $(0.122 \mathrm{~Hz}$ and $0.0625 \mathrm{~Hz}$, respectively); in (D) and (E) the oscillation frequency was high $(2.44 \mathrm{~Hz}$ and 4 $H z$, respectively.) The oscillation amplitude, $A$, was $\pm 6^{\circ}$. (B) and (D) show the visually induced yaw torque responses (upper trace) in experiments where figure and ground were initially oscillated synchronously and were then set to a relative phase of $90^{\circ}$. The stimulus traces at the bottom of the figures indicate the deviation of figure and ground from their mean position. Upward and downward deflections of the stimulus traces denote clockwise and counterclockwise motions, respectively. $(B)$ and $(D)$ represent averages from a total of 35 stimulus presentations in 7 flies, and 280 presentations in 14 flies, respectively. Positive and negative torques represent turning tendencies to the right and left side, respectively. The responses to synchronous and relative motion do not differ significantly at the low oscillation frequency. The fly mainly tries to follow the ground motion with a large response amplitude. At the high oscillation frequency during relative motion, flies try to turn towards the figure. This indicates that the figure is detected. Note that the torque responses to synchronous oscillation are much smaller at the high than at the low oscillation frequency. $(C)$ and $(E)$ show the response amplitudes to synchronous oscillation of figure and ground $(F+G)$ and to figure motion alone $(F)$. The data are averages from experiments with 25 (C) and 40 (E) flies (Musca). The response amplitudes were normalized with respect to the response induced by figure motion alone. At low oscillation frequencies, the largest responses are elicited by synchronous motion of figure and ground. In contrast, at high oscillation frequencies the largest response amplitudes are elicited by small moving patterns. (Part of data taken from Ref. 28).

analysis of the fly's visual system. From these experimental data, we extracted the underlying neuronal computations and formulated a model circuit that is sufficient to account for information processing tasks at both the neuronal and behavioural levels. Since turning about the vertical axis is a particularly important reaction component in visual orientation, the analysis was restricted to this degree of freedom. Whether the conclusions drawn here can be generalized to the other degrees of freedom is currently being investigated.

\section{Coherent and relative motion of objects and background are evaluated by two parallel systems with different temporal and spatial properties}

In free flight, the fly faces very complicated visual stimulus conditions which cannot be simulated and manipulated for experimental purposes. Our analysis was, therefore, done mainly on flying animals that were tethered to a torque meter and stimulated with moving patterns. In this way, the stimulus conditions could be exactly controlled. The visually induced yaw torque was measured as a good behavioural indicator of the fly's turning tendency. In a typical experiment, the test fly is positioned within a panorama of random dots (ground) which contains a small vertical stripe (figure) (Fig. 2A). The figure had the same texture as the ground, since the responses to relative motion rather than to different pattern characteristics were of interest. Both figure and ground were moved horizontally, either together or independently, thereby mimicking, respectively, coherent large-field and relative motions ${ }^{18}$.

Under these conditions, the figure is virtually invisible if it moves synchronously with the background. The fly only responds with optomotor turning reactions which, in free flight, would minimize the relative velocity between stimulus and eyes and thus stabilize its flight course. Hence, the yaw torque oscillates about the straight-ahead direction as is shown for two different oscillation frequencies on the left-hand side of Fig. 2B, D. The response amplitudes, however, are much smaller at high than at low oscillation frequencies. When figure and ground move relative to each other, the figure may, in principle, be distinguished. However, whether it is distinguished by the fly depends not only on the phase relationship of figure and ground motion, but also on the oscillation frequency of the pattern. When there is an appropriate phase shift between figure and ground motion (e.g. $90^{\circ}$ as in Fig. $2 \mathrm{~B}, \mathrm{D}$ ) and the oscillation frequency is high (between about $0.5 \mathrm{~Hz}$ and $8 \mathrm{~Hz}$ ), the time course and mean values of the response profiles change considerably (Fig. 2D). A mean torque response is generated towards the figure which, in free animals, would bring the figure in front of the eyes. This indicates that the fly has detected the figure and is trying to fixate it. In contrast, at low oscillation frequencies (below about $0.2 \mathrm{~Hz}$ ), neither the time course of the response to relative motion nor its mean value differ much from those elicited by coherent motion (Fig. 2B). Hence, the fly does not respond to the figure in any obvious way. Thus, for equally textured stimuli relative motion is necessary, but not sufficient for figure-ground discrimination: the figure can only be discriminated at higher oscillation frequencies.

There is now good behavioural evidence that the visually induced yaw torque responses are jointly mediated by at least two parallel, bilaterally symmetrical control systems. This conclusion has been derived from behavioural experiments on normal unimpaired flies ${ }^{20,28}$, and on flies after microsurgical lesioning ${ }^{29}$ or laser ablation ${ }^{30}$, of defined neuronal structures. It is consistent with what has been proposed for other fly species, such as syrphids ${ }^{13}$ and Drosophila $^{21,22}$. In house- and blowflies, the two control systems differ in: (1) their sensitivity to stimulus size and direction of motion; (2) their interocular interactions; and (3) their dynamical properties $^{18,20,28,29}$.

One of these control systems mediates yaw torque mainly at low oscillation frequencies and is more 
sensitive to the motions of large rather than small stimuli (Fig. 2C). This 'large-field' (LF) system appears to be essentially responsible for the optomotor compensation of retinal image displacements of the entire surround, and mediates course stabilization. In contrast, the other system dominates at high oscillation frequencies and is sensitive to small patterns (Fig. 2E). This 'small-field' (SF) system mediates the detection, fixation and tracking of small moving objects. Both systems can be activated by monocular stimulation and are affected in different ways by simultaneous motion in front of the contralateral eye; whereas the SF system is inhibited by contralateral motion, the LF system can be activated by such stimulation. These interactions enhance the sensitivity of the LF and SF systems to global rotational motion patterns and object motion, respectively.

The different sensitivities of the LF and SF systems to the size and dynamics of the stimulus are an important determinant of the behavioural response adopted by the animal under free-flight conditions. Slow changes of the direction of retinal image motion may result from external disturbances or asymmetries in the flight motor. These unintended deviations from the flight course are compensated for by corrective steering manoeuvres dominated by the LF system. Active turns, on the other hand, may not be counteracted by the LF system. This is because houseflies do not turn smoothly in free flight when purposely changing course, but instead make several rapid turns ${ }^{16}$. Under these conditions, the LF system is relatively inactive, while the SF system remains operational ${ }^{28}$. This suggests that under natural conditions, the two systems will not interfere much with one another owing to their different dynamic and spatial properties. Thus, they seem to be highly adapted to extract from complex retinal motion patterns the information relevant to course stabilization and the detection and fixation of objects. Of course, the problem of how to separate the visual consequences of active and unintended turns is not specific to the fly; it is encountered whenever an animal moves around in a structured environment. Although the solution to this problem as evolved in the fly is strikingly simple and elegant, it remains to be seen whether it is also adopted by other species.

\section{The neuronal basis of the LF and SF systems}

Each compound eye of the fly scans about one hemisphere of the environment. The main projection from the retina to the brain is through three consecutive retinotopically organized visual ganglia ${ }^{31}$ (Fig. 3A), Along this pathway, extensive transformation of the input information occurs $^{32}$. The point-to-point representation of visual space is abandoned in the posterior part of the third visual ganglion, the lobula plate. Here, the information is spatially integrated by about 50 large interneurones, each of which scans either the entire visual field or particular sections of it. Some of these interneurones connect to the contralateral lobula plate, others to descending neurones ${ }^{24}$. The latter project directly, together with input from other sensory modalities, to the motor control centres in the thoracic ganglia ${ }^{33,34}$.

It is mainly in the lobula plate that the processing of global motion patterns received by the eyes during flight manoeuvres occurs ${ }^{24}$. The extraordinary structural constancy and highly invariant physiological characteristics of its different cells allows them to be identified individually in each animal. All cells of the lobula plate investigated so far are activated by motion in a particular preferred direction, and are inhibited by motion in the opposite direction. The preferred directions are aligned either with the horizontal or vertical axes of the eyes. The neurones are presumed to receive input from local EMDs. Two types of lobula plate output elements play a decisive role in yaw torque generation. They are sensitive to horizontal pattern motion, and are likely to represent the cellular analogues of the LF and SF systems, respectively. These are the horizontal cells ${ }^{25,26}$ and the figuredetection cells ${ }^{27}$.
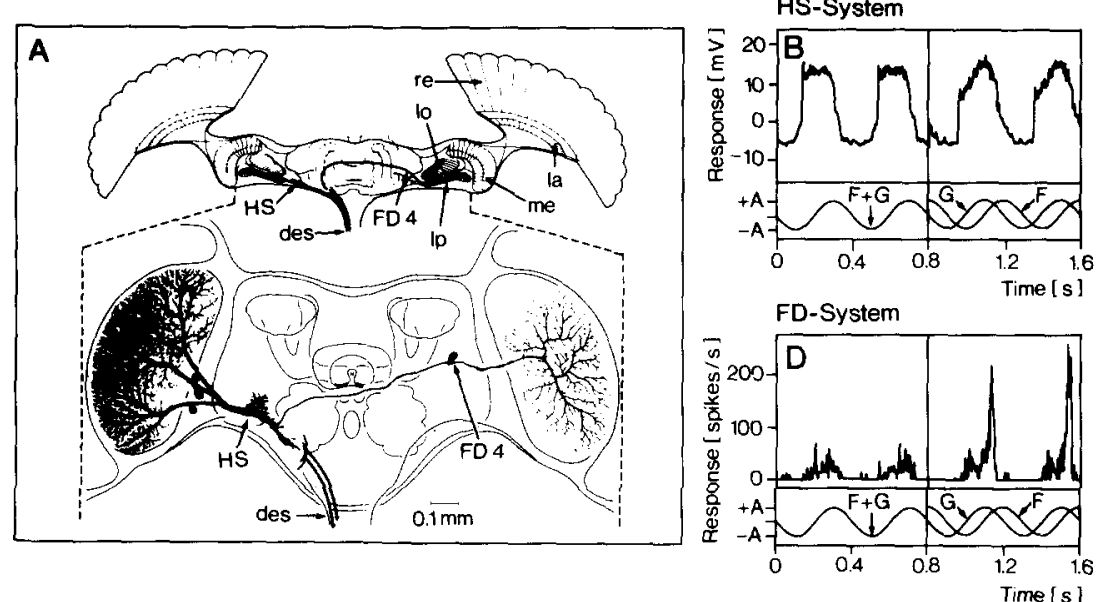
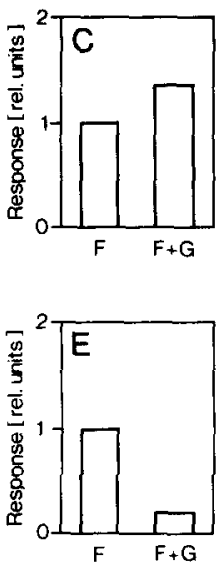

Fig. 3. Neuronal components of the LF and SF systems. (A) Upper diagram: schematic diagram of a horizontal cross-section through the compound eyes and optic lobes of the fly. The ommatidia in the retina (re) and the corresponding columns in the visual ganglia (la, lamina; me, medulla; lo, lobula; (p, lobula plate) are schematically indicated. In the lobula plate, the retinotopic information is spatially integrated by the large horizontal cells (HS) and figure-detection cells (FD). They project into the central brain and are connected via descending neurones (des) to the thoracic motor centres (not shown). Lower diagram: anatomical structure of the three HS cells and the FD4 cell as revealed by cobalt and Lucifer Yellow stainings. (B, D) Time-dependent responses of an HS and an FD4 cell to both coherent large-field and relative motion. The stimulus was essentially the same as in the corresponding behavioural measurements (see Fig. 2). The oscillation frequency and amplitude, $(A)$, were $2.5 \mathrm{~Hz}$ and $\pm 5^{\circ}$, respectively. The figure had a width of $10^{\circ}(B, C)$ or $24^{\circ}(D, E)$. The mean position of the figure was in front of the right eye at an angular position of $+40^{\circ}(B)$ and $+60^{\circ}(D)$. All data were obtained with Calliphora. The responses of the HS cell to stimulation with synchronous oscillation of figure and ground and relative motion with a phase shift of $90^{\circ}$ show only minor differences. In contrast, the FD4 cell shows only weak responses to coherent large-field oscillation and a characteristic sharp response peak during relative motion. The HS responses are averages of the cell's graded membrane potential changes obtained from 20 stimulation sequences. in contrast, the FD4 cell responses represent spike frequency histograms averaged from 16 stimulus repetitions. (C, E) Averaged peak responses of the HS and FD4 cell to synchronous oscillation of figure and ground $(F+G)$ and figure oscillation $(F)$. The response amplitudes were normalized as in the corresponding behavioural experiments (see Fig. 2). The data are averages of 20 (C) and 120 (E) stimulus cycles. The HS cell shows its largest responses to coherent large-field motion; the FD4 cell to the movement of a small figure in its excitatory receptive field. (Data from Refs 18,27 ). 

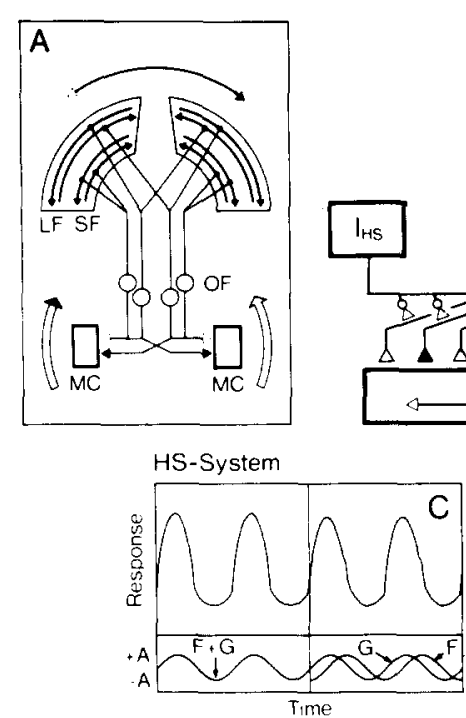

B
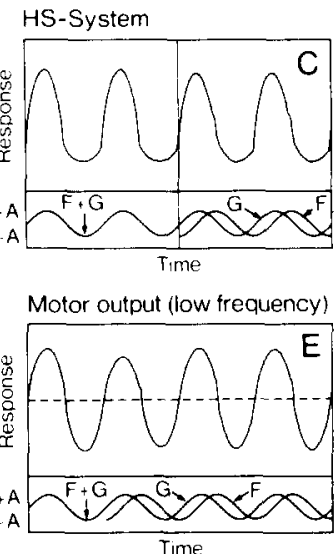

\section{(1)}
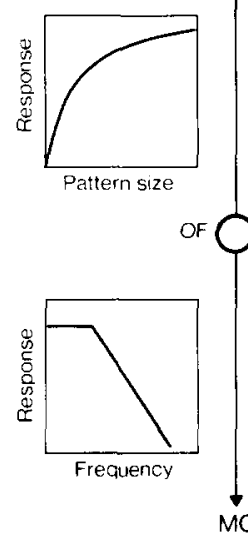

Fig. 4. Possible mechanisms accounting for the spatial integration properties of the LF and SF systems. (A) Schematic outline of the neural circuits controlling motion-induced yaw torque responses. Horizontal motion activates two functionally distinct types of control systems behind each eye that integrate the output of retinotopic arrays of EMDs. They are either tuned to $L F$ or $S F$ motion. The arrows indicate that the $L F$ system is activated by ipsilateral motion from front to back and contralateral motion from back to front, and induces yaw torque responses by excitation and inhibition of the appropriate contra- and ipsilateral control systems of the flight motor (MC). The SF system is activated by ipsilateral horizontal motion of small objects in either direction and mediates turning responses towards the stimulus. (Solid and open arrowheads indicate a positive and negative sign, respectively). The output channels of the LF and SF systems are frequencyfiltered in a different way (OF). In (B) a model circuit representing components of the $L F$ and $S F$ systems in the right optic lobe shows part of these interactions. The input elements are retinotopically arranged EMDs (abbreviations not listed in Fig. 1: $R$, receptor; F, low-pass filter; and $M$, multiplication). Their outputs are spatially summated by the HS and FD cells. One of the output branches of each detector is excitatory (-), the other is inhibitory (- $\longrightarrow$ ). This gives the cells their directional selectivity. Prior to their summation, the EMD outputs are inhibited, via synapses of the shunting type, by other large-field elements $\left(I_{H S}\right.$ and $\left.I_{F D}\right)$. I $I_{H S}$ shows monocular sensitivity to ipsilateral horizontal motion in both directions, whereas $I_{F D}$ is sensitive to horizontal motion in front of both eyes. The strength of shunting inhibition depends on the response amplitude of $I_{H S}$ and $I_{F D}$ and, thus, on the size of the moving stimulus. The spatial integration properties of both systems depend on the transfer characteristics of the output synapses of the EMDs. If appropriately chosen, the output of the HS system increases with increasing pattern size, while the response of the FD system decreases after reaching its maximum (see insets). (C) and (D) show the time course of the HS and FD cell responses to synchronous and relative oscillatory motion as simulated on the basis of this model network. The stimulus traces below the figure have the same meaning as in the corresponding electrophysiological experiments. (E) and (F) show the simulated torque responses to synchronous and relative motion at two oscillation frequencies. As in the corresponding behavioural experiments, the relative phase between figure and ground is switched to $90^{\circ}$ after one cycle of synchronous oscillation. Due to the different frequency transfer characteristics in the $L F$ and SF output channels, they contribute to the behavioural response with a different gain. In $(E)$ and $(F)$ the gain ratios of the $L F$ and SF systems amount to $10: 1$ and $1: 10$, respectively. The computer simulations of both the cellular and behavioural responses fit closely to the corresponding experimental results. (Model adapted from Refs 18, 35.)

The horizontal system (HS) consists of three neurones that scan the dorsal, medial, and ventral parts of the ipsilateral visual field and are accordingly termed north, equatorial, and south horizontal cells (HSN, HSE, HSS) (Fig. 3A). These cells project into the ipsilateral part of the brain and are synaptically coupled to descending neurones. All three horizontal cells are excited by motion from the front to the back within their receptive fields. Due to synaptic connec- tions from a large-field cell of the contralateral lobula plate, the HSN and HSE cells also respond to motion from back to front in the contralateral visual field ${ }^{25,26}$. This makes these cells particularly sensitive to rotatory motion about the animal's vertical axis.

The figure-detection (FD) cells are an anatomically heterogeneous group of at least four output cells of the lobula plate ${ }^{27}$. The FD4 cell, which will be considered here as a representative example of the FD cells, scans most of the ipsilateral visual field (Fig. 3A). The cell projects through the central brain into the contralateral visual centres. Although detailed connectivity studies are not available, it is likely that the FD4 cell is also coupled to descending neurones. The FD4 cell selectively responds to motion from the front to the back. Other FD cells are excited by motion from the back to the front ${ }^{27}$.

The typical response patterns of both HS and FD cells during stimulation with coherent large-field motion and relative motion are shown, respectively, in Fig. 3B and D. The stimulus conditions were virtually the same as in the corresponding behavioural experiments. Figure $3 \mathrm{~B}$ shows the response of the HSE cell to synchronous and relative oscillation of figure and ground. Synchronous front-to-back motion leads to activation (depolarization), and back-to-front motion leads to inactivation (hyperpolarization) of the cell. With relative motion of figure and ground, the response pattern changes only slightly. Thus, the influence of the moving figure on the transient response profile of the HS cells is small as compared with the influence of the background. This is reminiscent of the behavioural response at low oscillation frequencies (see above). The responses of the FD4 cell to the same stimulation paradigms differ considerably. As shown in Fig. 3D, synchronous front-to-back motion of figure and ground elicits only a small response. Relative motion of figure and ground with a phase difference of $90^{\circ}$ leads to significant response peaks. Although there is a phase shift owing to synaptic delays and transmission times within the nervous system, these response peaks have a similar time course to those given by the corresponding yaw torque responses at high oscillation frequencies and occur when the figure moves in a front-to-back direction and the ground motion is transiently zero. The response pattern indicates that the 
FD4 cell responds preferentially to the movement of small objects.

This characteristic difference between the HS and FD cells has been substantiated by measurements of the spatial integration properties of the two cell types $^{26,27}$. While the response of the HSE increases with stimulus size, the FD4 response reaches its maximum during stimulation with a small pattern (Fig. $3 \mathrm{C}, \mathrm{E})$. In other words, the HS and FD cells are selectively tuned to large-field and small-field motion, respectively.

We conclude from their specific response properties that the HS and FD cells represent the neuronal analogues of the LF and SF systems, respectively. The HS cells are specialized to evaluate global retinal motion fields as they are induced during rotatory selfmotion of the animal about its vertical axis. The outputs of these cells, therefore, signal course deviations and are used to control corrective flight torques. The FD cells, on the other hand, signal retinal image displacements of relatively small objects against the background. Their functional significance, therefore, could involve orientation towards objects.

A comparison of the dynamic properties of both cell classes with the behavioural responses suggests that high frequency modulations in the output signals of the HS cells are greatly attenuated somewhere between the lobula plate and the final motor output. Hence the HS system does not contribute much to the yaw torque at high oscillation frequencies, whereas the FD cells remain effective (Egelhaaf, M., unpublished observations). The relative contributions of each cell class to the final motor response thus varies according to the dynamic properties of stimulus motion (see above).

\section{Mechanisms for the spatial integration properties of the LF and SF systems}

Although the characteristic response properties of the HS and FD cells are still under investigation, the possible underlying computational mechanisms are constrained by theoretical considerations and network modelling ${ }^{18,35}$. The proposed computational models are plausible in terms of the neuronal hardware available in the fly's brain and, therefore, can be tested in further electrophysiological experiments.

Despite considerable differences in their spatial integration properties, the responses of the HS and FD cells can be demonstrated by the same type of model network, provided the appropriate model parameters are chosen ${ }^{18,35}$. A simplified version of the model circuit is shown in Fig. 4B. It consists of a retinotopic array of EMDs, inhibitory elements, and two groups of integrative directionally selective output elements. The latter correspond to the HS and FD systems. The inhibitory elements integrate the signals of the movement detectors, and subsequently inhibit them via synapses with an inhibitory equilibrium potential close to the resting potential (shunting inhibition). The strength of inhibition depends on the size of the moving stimulus. After the shunting operation, the output elements integrate the signals of the EMDs.

The input circuitries of the model HS and FD cells differ in two respects ${ }^{18,35}$ : (1) it must be assumed that the output terminals of the movement detectors have different non-linear synaptic transfer characteristics; and (2) the proposed inhibitory elements in the input circuitries of both cell types have different preferred directions and binocular sensitivities. If these characteristics are chosen appropriately ${ }^{18,35}$, the response of the simulated HS cell increases slightly as the number of stimulated movement detectors increases, whereas the model FD cell shows a pronounced response to small stimuli that decreases considerably as the stimulus pattern becomes larger (Fig. 4B). Moreover, on the basis of this type of circuit, not only can the different spatial integration properties of the HS and FD cells be modelled, but the network also reproduces the specific time courses of the cellular responses to relative motion between figure and ground (see Fig. 4C, D).

In a further step, the complex behavioural responses are reconstituted by the model responses of the HS and FD cells. As discussed in the last section, the $\mathrm{HS}$ and FD cells are considered as elements of the LF and SF systems (Fig. 4A). The two systems are assumed to interact additively, with only their gain depending on the stimulus conditions. If the relative contribution of the HS system to the final motor output decreases with an increasing oscillation frequency of the stimulus pattern, while the gain of the FD system stays large, the characteristic properties of the behavioural response profiles at low and high oscillation frequencies (see Fig. 2) can be easily obtained (Fig. 4E, F).

The close agreement of the computer simulations of the model circuit with the cellular and behavioural responses suggests that: (1) the cellular model is a plausible representation of the likely neural mechanism underlying the LF and SF systems; and (2) the properties of the HS and FD cells are sufficient to explain both visual course stabilization and the discrimination and subsequent fixation of small objects. Moreover, the proposed model network accounts not only for figure-ground discrimination by relative motion as has been analysed here; owing to the specific properties of the EMDs, the model also allows, without any further assumptions, the discrimination of an object from its background when they differ only in their textural properties ${ }^{36}$. The LF and SF networks thus detect both temporal and textural discontinuities within the complex retinal motion fields.

\section{Concluding remarks}

The results presented here show that in the fly, both visual course stabilization and orientation towards objects are achieved by the parallel computation of local motion in retinotopic arrays of movement detectors. This local motion information is subsequently processed in two subsystems that have different dynamic and spatial integration properties. These properties endow one system with sensitivity to rotatory motion of the entire visual scenery and the other to relative motion of objects and their background. At the neuronal level, the subsystems could be attributed to two types of output elements of the optic lobes, the HS and the FD cells. These two systems might be of importance beyond the detection and fixation of stationary and moving objects as measured in our behavioural paradigms. Relative motion between objects and their backgrounds might also be the decisive visual cue in other tasks that require information on the three-dimensional structure of the visual surround, such as the avoidance of 
Acknowledgements We are grateful to $A$. Borst and J. Raper for critically reading the manuscript. in

particular, J. Raper made valuable suggestions for improving the

English. We thank

L. Heimburger and

1. Geiss for drawing the figures and for secretarial assistance. obstacles and predators, or the selection of appropriate landing sites.

Evaluation of coherent large-field and relative motion appears to be of general relevance in visual information processing. This is demonstrated by the fact that separate mechanisms for extracting these types of global retinal motion patterns have been found in a variety of different visual systems. These comprise other insects ${ }^{23,37-39}$ as well as different vertebrate species ${ }^{40-43}$, including $\operatorname{man}^{44-46}$. One example may suffice to substantiate this notion. Neurones with response properties reminiscent of the FD cells have recently been found in the middle temporal region (area MT) of monkey cortex ${ }^{42,43}$. Since lesions of area MT lead to deficits in the ocular tracking of small targets, these cells have been proposed to be involved in control of eye movements ${ }^{47}$ and thus might serve related purposes to the FD cells. On the other hand, physiological and anatomical studies in a variety of vertebrates have consistently pointed to the importance of the accessory optic system in processing visual large-field motion leading to compensatory eye and head movements ${ }^{40}$. This brain area might thus be functionally analogous to the HS cells. These similarities indicate common strategies throughout the animal kingdom for extracting different motion cues, and thus substantiate the importance of using comparatively simple systems, such as the fly's visual system, to elucidate the neuronal mechanisms underlying this type of computational task.

\section{Selected references}

1 Gibson, J. J. (1950) The Perception of the Visual World Houghton Mifflin

2 Koenderink, J. J. (1986) Vision Res. 26, 161-180

3 Reichardt, W. (1986) Vision Res. 26, 113-126

4 Buchner, E. (1984) in Photoreception and Vision in Invertebrates (Ali, M. A., ed.), pp. 561-621, Plenum Press

5 Reichardt, W. (1961) in Sensory Communication (Rosenblith, W. A., ed.), pp. 303-317, MIT Press

6 Reichardt, W. (1987) J. Comp. Physiol. A Sens. Neural. Behav. Physiol. 161, 533-547

7 van Doorn, A. J. and Koenderink, J. J. (1983) IEEE Trans. Syst. Man Cybern. 13, 916-922

8 van Santen, J. P. H. and Sperling, G. (1984) J. Opt. Soc. Am. A1, 451-473

9 Buchner, E. (1976) Biol. Cybern. 24, 85-101

10 Egelhaaf, M. and Reichardt, W. (1987) Biol. Cybern. 56, 69 87

11 Land, M. F. and Collett, T. S. (1974) J. Comp. Physiol. 89 331-357

12 Collett, T. S. (1980) J. Comp. Physiol. 138, 271-282

13 Collett, T. S. (1980) J. Comp. Physiol. 140, 145-158

14 Wehrhahn, C., Poggio, T. and Bülthoff, H. (1982) Biol. Cybern. 45, 123-130

15 Wagner, H. (1986) Philos. Trans. R. Soc. London Ser. B 312 , 553-579

16 Wagner, H. (1986) Philos. Trans. R. Soc. London Ser. B 312 , 581-595

17 Reichardt, W. and Poggio, T. (1976) Q. Rev. Biophys. 9, 311-375

18 Reichardt, W., Poggio, T. and Hausen, K. (1983) Biol. Cybern. 46 (Suppl.), 1-30

19 Wehrhahn, C. and Hausen, K. (1980) Biol. Cybern. 38, 179 186

20 Egelhaaf, M. (1985) Biol. Cybern. 52, 123-140

21 Heisenberg, M. and Wolf, R. (1984) Vision in Drosophila, Springer-Verlag

22 Bausenwein, B., Wolf, R. and Heisenberg, M. (1986) J. Neurogenet. 3, 87-109

23 Collett, T. and King, A. J. (1974) in The Compound Eye and Vision of Insects (Horridge, G. A., ed.), pp. 437-466
24 Hausen, K. (1984) in Photoreception and Vision in Invertebrates (Ali, M. A., ed.), pp. 523-559, Plenum Press

25 Hausen, K. (1982) Biol. Cybern. 45, 143-156

26 Hausen, K. (1982) Biol. Cybern. 46, 67-79

27 Egelhaaf, M. (1985) Biol. Cybern. 52, 195-209

28 Egelhaaf, M. (1987) J. Comp. Physiol. A Sens. Neural Behav. Physiol. 161, 777-783

29 Hausen, K. and Wehrhahn, C. (1983) Proc. R. Soc. London Ser. B. 219, 211-216

30 Geiger, G. and Nässel, D. R. (1982) Biol. Cybern. 44, 141149

31 Strausfeld, N. J. (1976) Atlas of an Insect Brain, SpringerVerlag

32 Laughlin, S. B. (1984) in Photoreception and Vision in Invertebrates (Ali, M. A., ed.), pp. 457-481, Plenum Press

33 Strausfeld, N.J., Bassemir, U., Singh, R. M. and Bacon, J. P. (1984) J. Insect Physiol. 30, 73-93

34 Heide, G. (1983) in Biona Report (Nachtigall, W., ed.), pp. 34-52, Akademie der Wissenschaften und der Literatur zu Mainz and Gustav Fischer Verlag

35 Egelhaaf, M. (1985) Biol. Cybern. 52, 267-280

36 Reichardt, W. and Guo, A. (1985) Biol. Cybern. 53, 285-306

37 Palka, J. (1972) Am. Zool. 12, 497-505

38 DeVoe, R. D., Kaiser, W., Ohm, J. and Stone, L. S. (1982) J. Comp. Physiol. 147, 155-170

39 Olberg, R. M. (1981) J. Comp. Physiol. 141, 327-334

40 Simpson, J. I. (1984) Annu. Rev. Neurosci. 7, 13-41

41 Frost, B. J., Scilley, P. L. and Wong, S. C. P. (1981) Exp. Brain Res. 43, 173-185

42 Allman, J., Miezin, F. and McGuinness, E. (1985) Perception $14,105-126$

43 Tanaka, K. et al. (1986) J. Neurosci. 6, 134-144

44 van Doorn, A. J. and Koenderink, J. J. (1982) Biol. Cybern. 44 167-175

45 Baker, C. L., Jr and Braddick, O. J. (1982) Vision Res. 22 851-856

46 Regan, D. (1986) Vision Res. 26, 127-145

47 Newesome, W. T., Wurtz, R. H., Dürsteler, M. R. and Mikami, A. (1985) J. Neurosci. 5, 825-840

\section{NINCDS Seeks Grant Proposals}

Several divisions of the National Institute of Neurological and Communicative Disorders and Stroke (NINCDS) are seeking grant applications.

The Division of Communicative and Neurosensory Disorders invites applications to study dysphagia associated with neurological disorders, and progressive hearing impairment.

For further information about dysphagia applications, contact Dr Judith Cooper, Division of Communicative and Neurosensory Disorders, NINCDS, Federal Building, Room IC06, 7500 Wisconsin Avenue, Bethesda, MD 20892, USA (telephone: (301) 496-5061). For further information on progressive hearing loss proposals, contact Dr Ralph Naunton in Room IC11 at the above address (telephone: (301) 496-1804). 